\title{
Applying Lean to Healthcare Delivery Processes - a Case-based Research
}

\author{
D.T. Matt ${ }^{\#}$, G. Arcidiacono*, E. Rauch ${ }^{\#}$ \\ ${ }^{\#}$ Faculty of Science and Technology, Free University of Bolzano (FUB), Piazza Università 5, Bolzano, 39100, Italy \\ E-mail:dominik.matt@unibz.it,erwin.rauch@unibz.it \\ * Dept. of Innovation and Information Engineering, Marconi University, Via Plinio 44, Rome, 00193, Italy \\ E-mail: g.arcidiacono@unimarconi.it
}

\begin{abstract}
The purpose of this paper is to demonstrate that Lean principles and methodology should be applied on a regular basis to the entire process flow of healthcare delivery systems. With reference to an actual case-based research, this article demonstrates how patient flows can be successfully optimized if Lean is not limited to single processes/contexts only, and it is applied to achieve holistic process improvement of an entire system. The complexity of healthcare delivery systems requires inclusive investigation from various points of view. This is why case-study-based research has been used to investigate dynamic, experiential and complex processes and areas, such as the ones featured by this article. The methodological basis for this research has been a twelve-step optimization approach outlined by the authors during a previous successful Lean programme. This same approach has been applied to optimize patient flows in the emergency departments of four different hospitals in Northern Italy. The research has involved teams composed of medical, nursing, technical and administrative staff. The results outlined in the article suggest that inclusive application of Lean tools leads to effective process optimization and a better working environment, when in connection with a systematic and holistic optimization approach. Feedback from participants was obtained through a satisfaction survey and a project assessment; it reported enthusiastic project acceptance and good teamwork climate. Among the results of the research performed in the four hospitals, several measures have been effectively implemented to reduce the lead-time for patients from registration to discharge. At the same time, patient-staff ratio and quality of care have been either maintained or even improved. However, lack of a definite conclusive evaluation can be explained by the research project still being implemented. The value of this paper lies in demonstrating how Lean contributes to achieve better process performance and high staff satisfaction, when implemented within the whole supply chain of a healthcare delivery system on a regular basis.
\end{abstract}

Keywords — Healthcare; healthcare delivery process; Lean; emergency department; patient flow improvement.

\section{INTRODUCTION}

In the last few decades, many healthcare organizations worldwide have been requested to do more with less resources. The overwhelming question has then become: could this be achieved without reducing both quality and quantity of services delivered to patients? Lean [1] has answered successfully, proving to be effective in overcoming such critical performance gap.

In Italy, healthcare heavily affects public budgets, due to its significant costs, and reducing "muda" (waste) can generate substantial savings. However, very often so far, Lean has been used to focus only on isolated processes and projects. On the contrary, this paper, in line with recent research [2], vouches for the need of Lean principles to be applied to entire complex systems as an integrated holistic approach (already applied by the authors in [3]) in order to achieve long-lasting substantial results. To eliminate the main causes that make healthcare costs increase, process inefficiency needs to be measured, and, consequently, reduced by implementing corrective actions - all this without considering factors that cannot be controlled, e.g. the age of population. Some inefficiencies are likely to be caused by clinical or by medical processes; others can be connected to administrative, logistics, and operational activities.

In this scenario, continuous improvement lies at the heart of Clinical Governance - the new healthcare management system introduced in the U.K. at the end of the 1990s, and the core project system around which Italian healthcare has been redesigned. The aim of a virtuously applied Clinical Governance is to grant each patient the best healthcare service, with the least amount of wasted resources, and with the utmost patient satisfaction. Features of the ideal 
healthcare system are indeed safety, efficacy, patientcenteredness, timeliness, efficiency, and equity. Such improvement of healthcare quality needs a structured approach to be obtained, and a development model involving patients, practitioners and the entire system as stakeholders.

If the entire healthcare supply chain was trained in Lean principles, it could benefit both from a shared language and from a common organizational framework of reference. Based on on-site research in Italian hospitals and on recent case studies on Lean Six Sigma [4] interventions in several healthcare contexts (e.g. [5]-[8]), this paper aims to demonstrate how Lean can be an effective instrument to improve the performance of healthcare delivery processes.

This paper presents a case study carried out using Lean principles to redesign the service delivery processes of the emergency departments in four Italian hospitals. The novelty of this paper lies in vouching for a holistic Lean approach on healthcare processes. Case study was considered the most appropriate applied research methodology to demonstrate how Lean principles, when implemented into the entire system, contribute to increase patient satisfaction, quality of care, working conditions for employees, and to lower the costs of the healthcare system.

The lessons learned from this case study highlight the positive impact of applied Lean methodology - its effectiveness on hospital system management and on process optimization.

The structure of this paper is as follows. Section 1 presents the literature review on Lean and the research objectives of this paper. Section 2 outlines core ideas and principles of Clinical Governance and Lean relevant to this study. Furthermore, this Section describes the research methodology used in this work. Section 3 describes the results of this study based on a case study in an emergency department. After a first definition and analysis of patient families a twelve-step approach is shown to optimize delivery processes of emergency departments in Healthcare. The authors describe seven already implemented steps in the cases study project. Results are described, and the implications and limits of the research are discussed. Finally, Section 4 provides conclusions and an outlook for further research.

\section{A. Literature review and research objectives}

Lean can be defined as "an integrated operational and sociotechnical approach of a value system, whose main objectives are to maximize value and thus eliminate waste, by creating cumulative capabilities" [9]. In 1990, James P. Womack, Daniel T. Jones and Daniel Roos, researchers at the Massachusetts Institute of Technology, presented the results of a study in which they worked out the basic principles of Toyota's leading production system in terms of efficiency and quality [10]. They published the results of their study under the term "Lean manufacturing" and "Lean production" [11]. Several researchers like as in [12] and in [13] developed also concepts and approaches to assess the success and sustainability of Kaizen events. Due to the success of the approach in industrial production, methods and ways of thinking were successively transferred to other areas (among others maintenance, R\&D, management, construction). Essentially, Lean means creating customer value and avoiding - wherever possible - all non-valueadding activities [14].

The successful application of Lean, Six Sigma, and TRIZ [15]-[16], the problem solving method based on logic and data to accelerate the project team's ability to solve problems creatively, to industry processes has been extended to service industry, public administration, transport systems, as well as airport terminal systems [17]. Moreover, they are increasingly used in hospitals as in [18]-[20], due to the need for flexible methodologies to optimize healthcare systems and to improve process flow [21] by devising effective measurement plans [22]. These latter studies focused on creating patient-oriented and efficient healthcare services.

First developed as a production philosophy [23] and quality system, Lean has been successfully applied in the service industry, and introduced to healthcare delivery systems. This because the development of Lean involved a shift towards both customer value and waste reduction, including human behavioural aspects in close connection with its original operational features. In the last decade, some avant-garde hospitals in the United States, Australia, and Europe have begun to optimize their processes by applying Lean [24] and Six Sigma to handle growing problems in hospital management as in [25]-[28]. Indeed, the interest in operations research applied to healthcare keeps being on top of the agenda [29], as well as most recent developments and application of continuous improvement [30]. Many case studies show a significant improvement in quality and efficiency through the introduction of Lean. Sanders and Karr [31] demonstrated how the quantitative power of Six Sigma and the speed of Lean can work together to improve the blood sampling process for a 1,000-bed tertiary care teaching hospital. Lean helped to decrease the number of vials used for testing up to a 50 per cent, and to decrease the time needed for the complete blood count up to a 30 per cent. Abdelhadi [32] applied on a case study the concept of takt time as a measure to compare the efficiency of two emergency departments, in order to assess service quality. Studies show that Lean is applicable to large, small and medium hospitals, and size is not relevant as to the amount of potential advantages gained [33]. Furthermore, according to [34], Lean seems to have a positive influence on employee satisfaction and on the reduction of absenteeism.

While it is encouraging that the ideas of Lean have been enjoying increasing interest in healthcare studies as well [35], lessons learned from the approaches reported in the literature so far have exploited a very limited amount of its actual achievable potential [36]. Indeed, Brandao de Souza [37] analysed over 90 publications from 2002 onwards referring to the use of Lean in healthcare. Reference [38] analyzed 177 articles on Six Sigma and Lean published between 2000 and 2008: 70\% were related to Six Sigma, $23 \%$ to Lean, and $7 \%$ only to both. Moreover, the literature review shows that those healthcare organisations implementing Lean mainly use only narrow-defined limited pilot projects and simple methods and tools. Reference [39] analyzed how Lean has been implemented in English hospitals. According to their findings, Lean implementation tends to be isolated rather than applied on the entire system. 
Dissemination strategies are important for a sustainable success. Reference [40] shows one possible approach for dissemination in multiple healthcare organisations: forming clear visions and objectives; piloting; training potential adopters; and formal dissemination. In her survey of 223 hospitals in New England, [41] investigated the relationship between Lean implementation and organizational culture. According to her results, group culture and management support are highly important. Thus, she suggested that hospital managers implementing a Lean Six Sigma initiative would be well served to use an approach and strategies that are consistent with group and developmental cultures. Furthermore, in their systematic literature review, Crema and Verbano [42] showed that Lean has often been used for cost-cutting, while having a significant impact on risk management in healthcare. Therefore, their suggestion is to develop integrated methodologies that include both Lean and risk management tools and practices. In support of this, in their review of seven literature reviews, [43] shows how Lean and Six Sigma management techniques have been implemented in acute care settings identifying that they have a positive impact, especially where processes involve a linear sequence of events.

However, the application of Lean and Six Sigma in healthcare has often led to resistance, mainly due to the objection that these concepts tend to disregard healthcare unique sociotechnical aspects [44], intended as the interaction between human behaviour and technical elements [45]. Moreover, some studies have found out a tendency to focus mostly on positive results only, when debating applications of Lean and Six Sigma [46]. According to [2], evidence about its contribution to higher organisational performance remains inconsistent. It must be said, though, that very few studies concentrate on negative outcomes of applied Lean [47]. However, despite a tendency in literature reviews [48], and in line with research on positive perception of applied Lean principles within healthcare systems [49], this paper aims to demonstrate that Lean principles, when applied to optimize patient flows, not only improve patient flows, but they also increase patient satisfaction and safety. Through methodological and practical reference to a hospital-based case study, it will discuss how implementing, and making them sustainable, Lean and Six Sigma principles [50] serve as effective holistic optimization of the hospital system.

Therefore, the research question of this study can be summarized as follows: How can Lean and Six Sigma help to improve performance, patient satisfaction and staff satisfaction by applying these methods systematically and holistically in healthcare operations?

To answer this research question, the following theories and hypotheses are to be studied and examined in this case study research:

- H1: Staff satisfaction can be sustainably improved by involving people and interdisciplinary teams in Lean projects.

- H2: A Lean approach increases customer value and, therefore, patient satisfaction.

- H3: Lead-time from registration to discharge can be minimized by applying Lean principles.
- H4: Lean should be implemented holistically in hospital structures and not just inside pilot projects.

Working on these hypotheses, this paper will disclose how most promising the combination of Lean and Six Sigma is in the context of healthcare delivery processes [51], and within the assessment of system quality management in a hospital.

\section{MATERIAL AND METHOD}

\section{A. Lean and Clinical Governance}

Clinical Governance and Lean were applied to successfully optimize patient flows; this same topic has been also studied by the authors in a previous study [52]. The aim was the holistic optimization; results were obtained through case-study based research on clusters of different projects developed inside the hospitals involved.

A study of the Juran Institute highlighted how 30\% of healthcare related costs in the U.S. were connected to poor quality. Ideally, thinking in terms of Clinical Governance, the so-called 4Es (Efficiency, Economy, Effectiveness, Efficacy) rule the organization through sharing and standardizing best practices. Therefore, Clinical Governance needs a strong commitment at all levels, which means working to:

- reach out for and understand patients' expectations;

- develop and maintain a management system capable of satisfying patients' needs in a reliable, repeatable, and cost-effective way;

- design services which mirror customers' (i.e. patients') needs;

- provide services mirroring what they have been designed for;

- verify that services have the requested features before they are provided;

- prevent the delivery of services which are potentially dissatisfactory for patients;

- isolate and eliminate unnecessary features of services to be provided;

- find more cost-effective operational solutions while keeping the standard of the required quality;

- make operational activities more efficient and effective;

- $\quad$ isolate, and implement, those features of services to be delivered that induce patients' utmost satisfaction.

Such mandatory goals are attainable by applying Lean to integrate the aims of Clinical Governance and those of continuous improvement. This because they share the efficacy-oriented goals applied to good clinical practice, error prevention, the optimization of available resources, and not only. Moreover, Lean could provide the necessary tools to reach the objectives described in the pillars of the Clinical Governance, as shown in Table 1 below.

This tight connection of Lean Six Sigma (LSS) and Clinical Governance justifies the success of such methodologies, which are no longer restricted to industrial processes; rather, they are applied as everyday tool for continuous improvement of all processes. Specifically, Lean has been applied most consistently to [19]: 
- $\quad$ reduce waiting times and delays (directly linked to process faults);

- increase hospital capacity and performance (costs, quality and timeliness of medical aid);

- $\quad$ reduce hospital stay time for intensive care patients;

- reduce errors connected to the document flow (hospitalization, suppliers' billing, etc.);

- $\quad$ reduce preparation time for medication and surgery;

- increase overall patient satisfaction.

TABLE I

EXAMPLES OF WASTE IN THE HEALTHCARE SYSTEM

\begin{tabular}{|c|c|}
\hline Waste Categories & Example \\
\hline Overusage & $\begin{array}{l}\text { - Excessive hospitalization } \\
\text { - Unneeded treatments (they entail } \\
\text { expense, without benefiting patients) }\end{array}$ \\
\hline Underusage & $\begin{array}{l}\text { - Lack of adequate timely treatment } \\
\text { - Failure to satisfy medication } \\
\text { demand (vaccines, tests, etc.) } \\
\text { - Cutting post-operative treatments }\end{array}$ \\
\hline Misusage & $\begin{array}{l}\text { - Patients put on wrong medications } \\
\text { - Wrong diagnoses } \\
\text { - Wrong use of machines } \\
\text { - Wrong post-operative treatments }\end{array}$ \\
\hline Waste & $\begin{array}{l}\text { - Operational bureaucracy } \\
\text { - Batch working (extending lead time) } \\
\text { - "Re-", "double" (doing things twice, e.g. } \\
\text { rework, double-check, etc.) }\end{array}$ \\
\hline
\end{tabular}

Previous experience in applying Lean to healthcare has highlighted how the most significant and successful improvements in healthcare services have been attained by extending the application of the methodology to all processes and to all inefficiencies detectable within the service supply chain.

High costs and scarce efficiency of the healthcare service are no longer tolerated, both by practitioners and by patients. Indeed, hospital managers are constantly working to reduce waste and hospital costs. Lean is a tool to support daily practices of continuous improvement, balancing patient care and scrupulous delivery of services to ensure their efficacy.

The case study under scrutiny in this paper allows to better understand the contribution that Lean methodologies can offer to the Italian healthcare system, and how they can be applied to everyday hospital practice at large.

\section{B. Research methodology in the case study}

Case study research using a single case study is particularly useful when a subject requires comprehensive investigation from multiple perspectives [53]. Single case study research in healthcare was adopted also by other researcher [35], [54], [55].

The case study reported in this article is taken from the authors' experience in a Lean programme, the aim of which was to develop a Lean-based holistic approach to achieve effective and long-lasting optimization strategies to be applied to diverse healthcare processes. Fig. 1 shows the applied research methodology in this Lean initiative.

The programme initiative, called "Lean Hospital" started with the definition of a long-term vision and strategy for implementing the Lean Hospital programme.
1. Lean Hospital Vision, Strategy and Masterplan [28]

- Axiomatic Design bascd development of a Lcan Hospital Vision and Strategy

- Elaboration of a Lean Hospital Masterplan with a detailed project plan
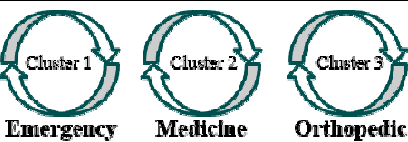

...
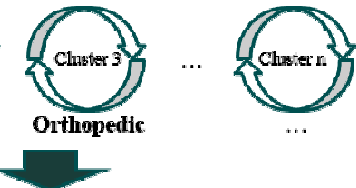

2. Lean Hospital Leadership

- Training of responsible and team leaders (train the trainer)

- Project marketing (presentations in the four case study hospitals)

- Communication through hospital intern newspaper/magazine

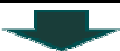

3. Training Workshops in Lean Management

- Theoretical training of all involved persons in Lean Management

- Practical exercises in the use of Lean tools

- First "sketch" of patient families based on professional experience

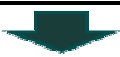

\begin{tabular}{|c|}
\hline $\begin{array}{l}\text { 4. Current State and Data Analysis } \\
\text { - Definition of patient families } \\
\text { - Analysis of patient flows (value stream mapping method) } \\
\text { - Data analysis of waiting times and bottlenecks }\end{array}$ \\
\hline $\begin{array}{l}\text { 5. Futwre State Mapping } \\
\text { - Application of I Ean lools } \\
\text { - Reorganization of processes } \\
\text { - Optimization of processes through Kaizen activities }\end{array}$ \\
\hline $\begin{array}{l}\text { 6. Implcmentation and Monitoring } \\
\text { - Scheduling of measures } \\
\text { - Implemcntation of mcasurcs } \\
\text { - Monitoring of measures }\end{array}$ \\
\hline
\end{tabular}

Fig. 1 Research methodology applied to the case study analysed in this article

Axiomatic Design was used as theoretical and methodological ground applied to the research, which resulted in a complete Masterplan outlined, with a detailed programme and project plan. The Axiomatic Design approach used in this phase is described in [28]. The project plan is long-term oriented and includes several subsequent clusters of projects carried out in parallel in the four selected hospitals of this case study. Every cluster of projects focused on one specific patient flow. The first cluster started a couple of years ago as a pilot project, and, so far, it has concentrated on the redesign of the emergency department according to Lean principles. Also reference [35] adopted pilot research in order to test and optimize the Lean approach, before expanding it to other departments. Other clusters should extend the Lean programme to the medicine department, the orthopaedic department as well as surgery and other patient flows. The emergency department has been chosen by the hospital management due to actual problems with long lead and waiting times, as well as bottlenecks in staff capacity. The sequence of other clusters was defined based on the priorities of the hospital management.

In a second project phase, people from the hospital management, as well as the appointed team leaders, were trained in Lean principles. In line with what [35] suggests, an effort was made to communicate Lean practice principles to decision makers. The project was communicated through presentations to the emergency department staff of the hospital. Furthermore, communication of the project was also provided through the hospital magazine. This to avoid scepticism to spread among the teams, and to answer 
questions regarding the objectives and the planned implementation.

In a third phase, the project teams of the four hospitals were invited to a series of training workshops. These workshops were helpful to transfer Lean theoretical knowledge and to give participants the chance to exercise the main Lean tools, like Value Stream Mapping, and not only. In addition, these workshops served to the teams to elaborate their first proposals of possible patient families, according to their professional experience.

In the fourth phase, each project team analysed the patient flows of their hospital using Value Stream Mapping, and further workshops for the analysis of the seven types of waste were organized. Moreover, using existing data from the information systems, additional data analysis could be conducted.

Based on the analysis results of phase four, the fifth phase was used to define the future state of processes and structures. Therefore, processes were reorganized, Lean tools applied, and processes optimized.

In the sixth phase, the project team defined an implementation roadmap with a list of measures to be taken to achieve the projected future state. Usually, this phase needs a close collaboration with the hospital management, in order to define the prioritization of measures based on their duration, their costs and their qualitative as well as quantitative improvements. Eventually, monitoring should be implemented to control the status of implementation. Up to date, no final monitoring of the results has been implemented, since several measures are currently being implemented, and the digital tracking of relevant data needs to be clarified with the IT Department of the hospitals.

So far, observations have been carried out following the Lean training of the teams. It was asked how satisfied participants were with the training, and whether they would recommend the training. The results were indeed quite positive. $90 \%$ of the participants were satisfied or very satisfied, and nearly $100 \%$ have recommended their colleagues to participate to the Lean Hospital Initiative.

The optimization steps, which resulted as the outcome of the programme, are reported in Section 3.

\section{RESULTS AND DISCUSSION}

\section{A. The "Emergency Department" case study: definition of patient families}

Participants were assigned to teams according to an interdisciplinary criterion. The typical team consisted of the following medical and nursing staff: 1 chief physician or head of the emergency department, 1 nursing coordinator, 1 senior doctor, 1 nurse, 1 radiographer, 1 laboratory technician, 1 administrative person, and 1 quality manager. The research began with training the project team in Lean principles. They had never been involved in Lean projects before; therefore, an initial training workshop helped to understand Lean theory as well as the single Lean tools used in healthcare. Suitable "patient families" were defined as the first step of the research. First ideas of possible patient families were collected in the initial training workshops. However, the final definition of patient families was based on data analysis that either confirmed or rejected the idea of the project teams. When grouping incoming cases, all patients belonging to a particular patient family could go through similar processing steps. The workshop showed that all project groups had clear ideas on how to categorize their patients. The experience of the case study showed that patient families can be classified according to the two criteria "pathology" and "severity index" inside an emergency department (paediatric cases are treated separately). The currently used Italian Triage severity index differentiates between four classes of severity: red (lifethreatening); yellow (impairment of important life functions); green (minor injuries or non-life-threatening diseases); white (no urgency; should be treated by the general practitioner). Actually, red and yellow cases make up to $12 \%$ of the presenting patients. Within the criterion "pathology", a second classification was considered useful according to "trauma" and "non-trauma" patients.

For every patient family, the current-state flow was mapped (see example in Figure 2). Based on this currentstate patient flow, the target-oriented development of an optimized future-state patient flow was started. Twelve generally applicable steps have been defined to optimize patient flows in hospitals: some of them were selected to be applied to this case study, as extensively outlined below.

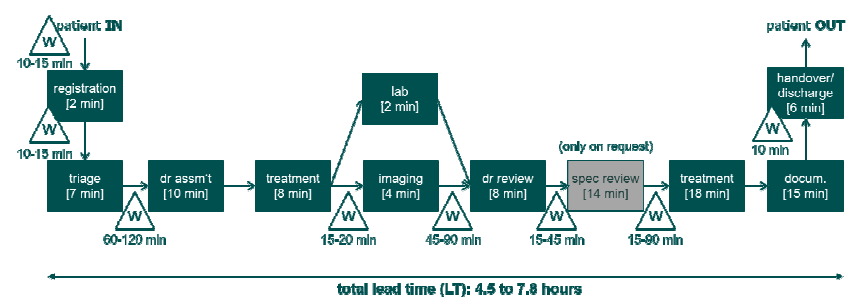

Fig. 2 Example of a patient flow in an emergency department

\section{B. Optimization steps}

Despite initial scepticism, the whole project team showed high commitment, being actively involved in workshops and working groups meetings. All team members were convinced of the performance and the benefits of Lean methods and tools, through its practical application when preparing positive proposals for improvement during working group meetings.

Among those, a twelve-step approach (originally adapted in [18]) used during a previously applied Lean programme, was applied in this case study as well.

The twelve-step approach has been arranged as follows:

1. Determine the required output rate (takt time) as an indication to determine the pace for every station in the process.

2. Create a continuous flow, balancing the station cycle times to the pace of incoming patients (takt time).

3. Implement visual control, and a fast intervention strategy to solve unplanned problems.

4. Introduce a workload optimized scheduling.

5. Reduce "set-up times" (preparation of rooms, instruments etc.).

6. Ensure an uninterrupted supply of defect-free input material by an initial quality check prior to process start. 
7. Use suitable standards and devices to prevent errors throughout the single processes in the patient flow.

8. Do not advance errors to the next station, or to the subsequent patient flow.

9. Eliminate or reduce non-value-adding activities.

10. Enable (medical and nursing) staff to operate more than one station.

11. Ensure flexibility to accommodate capacity increase at lowest cost.

12. Minimize one-time expenditures by investing preferably in modular system components.

Due to a prioritization of the activities proposed by the teams themselves, only the steps 1-4 and 9-11 have been implemented so far. The remaining five steps $(5-8,12)$ have been postponed, and are currently subject to optimization. Below, the outcomes of the implementation of these steps in the four emergency departments will be analysed, discussing and - where already quantifiable - evaluating their effectiveness.

1) Step 1 - Determine the required output rate (takt time) as an indication to determine the pace for every station in the process: The first optimization step required the definition of a takt time for the single patient flow. The analysis of data demonstrated that the patient input showed little variation over a year, with some minor peaks, as well as little variation over the week with some exceptions; however, data showed that it was very irregular over the day. During a 24-hour emergency service, about $80 \%$ of the flow occurs between $8 \mathrm{am}$ and $8 \mathrm{pm}$ (see Figure 3). Therefore, calculating an average takt time over 24 hours, based on this distribution, would not make sense.

\section{$\mathbf{8 0} \%$ of accesses between 8 am and $8 \mathrm{pm}$}

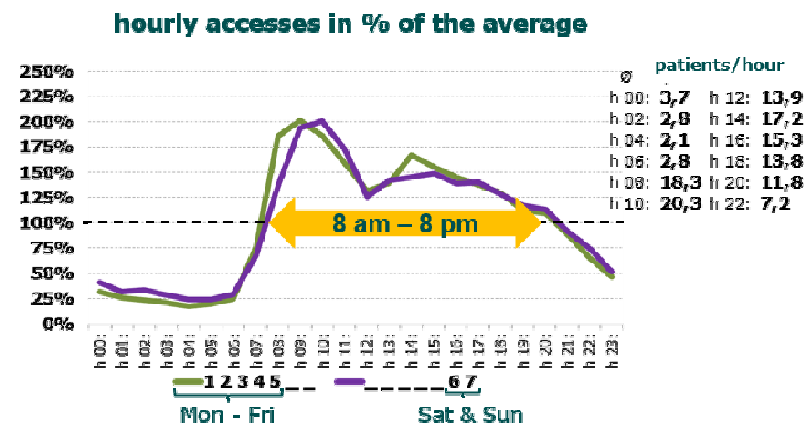

Fig. 3 Access variation to the emergency department over 24 hours

Therefore, two blocks of highly different access rates are detected: from $8 \mathrm{am}$ to $8 \mathrm{pm}$, an average of 221.2 patients come to the emergency department. In these twelve hours (=720 min), an average takt time of $3.2 \mathrm{~min}$ can be calculated. From $8 \mathrm{pm}$ to $8 \mathrm{am}$, however, the total access is only 37.2 patients, which leads to an average takt of 19.3 $\min$.

2) Step 2 - Create a continuous flow: Out of the presentstate patient flow, the actual processing times of the single stations can vary from 2 minutes to 18 minutes. In order to create a continuous flow, the cycle times of the single stations need to be aligned to the average takt time. Several measures (work packages) were defined to (a) reduce the single cycle times, and to (b) balance them to the takt time. One of these work packages, for example, had the objective to reduce cycle times and improve reliability in the triage station. Reliability means the coincidence of assessment results of different assessors in the triage system. It is useful because different human triage assessors will not make their judgements the same way. Therefore, a reliable triage system is given if different assessors make a uniform or similar judgement. In this case, we focused on consistency of ratings between different triage nurses. Reliability is given by means of $\kappa$-statistic: in a random result, $\kappa$ equals 0 , at $\kappa=1$ there is an absolute correspondence between $\geq 2$ measurements of different triage assessors. The following terminology has been used: poor $(\kappa<0.2)$, sufficient $(0.2<\kappa$ $<0.4)$, satisfactory $(0.4<\kappa<0.6)$, good $(0.6<\kappa<0.8)$, and very good $(0.8<\kappa<1)$ match [56]. The introduction of the 5-stage Manchester Triage System (MTS) brought an increase of the reliability factor from previously 0.67 to 0.82 , already after three weeks [27]. The increase in reliability is based on the introduction of systematic standardized protocols used in the triage assessment. Through a highly standardized approach in MTS the value for reliability could be increased as described before. It is expected that this figure will continue to rise, while being implemented on a broader scale, and to further reduce throughput times, increasing the quality and efficiency in the emergency department.

3) Step 3 - Implement visual control, and a fast intervention strategy to solve unplanned problems: Based on this optimization step, a "queue management system" was developed and implemented. The aim was to create perceptible transparency, order and process orientation in the emergency department. Previously, about $40 \%$ of patients did not know their classification (severity index) and had trouble finding the right waiting areas and understanding process sequences. This entailed a negative impact on the perceived quality of care and on patient satisfaction, first, and an additional burden on the medical and nursing staff. With the new "queue management system", patients know exactly where to go next, and can have an idea about their waiting time. Patients are guided through the hospital by visual management using signs and colours on the floor and walls. Thus, they can find the area they are looking for without stress, without the need to ask questions and to interfere with professional staff at work. Benchmark analyses were carried out by the working group in similar problem situations, such as in airports or in large shopping malls. For example, patients are guided to the right area via letter-number combination (like airport gates - e.g. Gate A1). To increase patient safety and to optimize the process, patients receive an identification bracelet. The identification bracelet is a strip of plastic that can be worn comfortably on the wrist. Information is displayed, based on which each patient can be immediately identified: name and surname, gender, date of birth, bar code. The latter is individual, and allows to clearly identify patients by electronic means, e.g. scanning. The data loaded on the bracelet are checked before each significant medical examination and/or operation. During this "check", patients are asked again their first and last name, and their date of birth. This information is then compared with the one registered on the bracelet. 
4) Step 4 - Introduce a workload optimized scheduling: The optimization of the staff workload was designed based on data gathered from the access curves (long-term average) in the four emergency departments involved in the research. Eventually, three different areas of activity were identified:

- Annual capacity planning: Based on collected and evaluated data, yearly access curves, annual assignments and holiday planning of the medical nursing staff has been analysed and improved. Depending on the statistically calculated peak, suggestions for an optimized workforce scheduling have been defined.

- Weekly planning of human resources: Based on the collected and evaluated distribution of patient access on the single weekdays, proposals for an improved staffing and scheduling have been developed, with the aim to provide better coverage for peak times (namely, Fridays and weekends) during the week.

- Daily planning of human resources: Based on the data collected and evaluated the daily access curve which follows the "elephant curve" shown in Figure 2, optimization measures were defined to be applied on personnel planning on a daily basis. Specifically, the precarious situation of the large number of access points in the late morning gave rise to adjustments of shift models and redeployment of resources. The curve showed potential high need of medical and nursing staff in the stressful time of the morning. This potential improvement was addressed through the employment of more part-time workers. On the one hand, this allows to cover the high peak in the morning; on the other hand, it allows employees to balance family and work, improving employee loyalty of qualified medical and nursing staff.

5) Step 9 - Eliminate or reduce non-value adding activities: New layout variants for remodelling the emergency department have been prepared by the project teams, to optimizing the layouts of the emergency services. The proposals were met with broad approval and support both by the medical and by the nursing staff. These new layouts allow a more efficient use of staff capacities, by shortening the distance between subsequent stations. Moreover, mixing elective and acute patient flows is avoided, with subsequently increased satisfaction of both patients and staff. The workgroup oriented itself towards an elaborated ideal layout for the emergency department, ensuring both optimal patient care as well as a reduction in the flow of people and materials (see Figure 4).

In this ideal layout, patients are immediately guided to a desk for registration. From there, they are guided through a quick triage, to a waiting room to await the treatment. Examination and Treatment rooms are located c lose to the waiting room: this reduces distances and allows the staff to handle several patients simultaneously. For serious cases, the emergency trauma room is positioned close to the entrance area, or near the access road and the elevator to the helicopter landing area. Further away there are doctors' offices for consultation and research, as well as for documentation work.

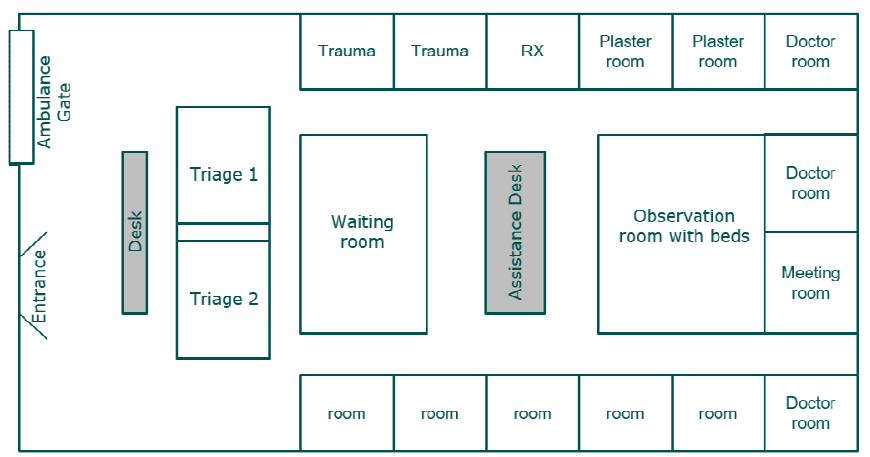

Fig. 4 Ideal layout for the emergency department

A space left unused has been redesigned for the preparation of medicines to improve the safety in the distribution of medicines and to increase the efficiency of preparation. While these were usually prepared on the station, a separate medication preparation room is now equipped with PCs and the latest technology.

6) Step 10 - Enable (medical and nursing) staff to operate more than one station: About the improved triage system, standards were introduced in close coordination and cooperation with the medical professionals, according to which now the nursing staff can order certain lab samples or start some treatment steps before the doctor's assessment. This leads to relieve the medical staff of some tasks, and to speed up the process. With a technical time of 45 minutes to process laboratory samples, this measure and the subsequent job enrichment for nursing staff allowed considerable time savings in the patient lead time. In addition, this also leads to greater patient satisfaction, saving some waiting time, as the nursing staff makes the decision for laboratory samples in autonomy without asking doctors. However, this change has required the introduction of a reliable and process-safe triage system (in this case MTS) and the definition of the corresponding protocols.

7) Step 11 - Ensure flexibility to accommodate capacity increments at lowest cost: To achieve a better adaptation to the actual capacity requirements, the shift models of the medical and nursing staff were made more flexible, and the capacity exchange between different medical stations was improved. Most patients in the emergency department have been admitted to the department of medicine. Through workshops with the medicine department and the emergency department, and the comparison of the utilization diagrams of the emergency and medicine, the capacity utilization between those could be optimized and synchronized. By means of a different scheduling of the documentation time of doctors, a sort of a stand-by service for bottleneck situations could be achieved at the emergency department.

\section{Results}

As previously outlined, the project and the implementation of the outlined activities are still ongoing. Therefore, intermediate results only can be reported so far; yet they are most encouraging. What follows is the discussion of the results to the research hypotheses formulated in Section 2. 
1) Staff satisfaction can be sustainably improved by involving people and interdisciplinary teams in Lean projects: A satisfaction survey conducted among all involved project participants showed that $90 \%$ of the participants were either completely satisfied, or very satisfied, with the initiative; almost $100 \%$ recommended their colleagues to participate in the Lean Hospital Initiative [28]. In addition, a subjective project assessment based on a more detailed feedback of each individual participant showed a high degree of acceptance of the Lean initiative and its related methods, despite initial scepticism of a significant part of the team members, especially among the medical staff.

2) A Lean approach increases customer value and, as a consequence, patient satisfaction: The hospital structure carries out patient satisfaction surveys at regular intervals. Through these surveys, on the one hand, a positive or negative trend is identified and, at the same time, "hot-spot" topics with particular interest for the patient can be detected. In the last satisfaction survey conducted before the Lean initiative, a negative trend in the patient satisfaction was noted. Particularly, the long waiting times for a medical consultation, long lead times in the emergency department, as well as insufficient time available for the nurses and doctors were complained. Since then, the Lean team has already implemented improvements to reduce the lead time, as well as to support the nursing and medical staff, which have led already to a better care for the patient. However, the next patient satisfaction measurement is still pending, so that quantitative data is currently not available.

3) Lead-time from registration to discharge can be minimized by applying Lean principles: For emergency departments, the lead-time for patients from registration to discharge (to a medical ward, to the medical assessment unit, or home) can be considered one of the most significant performance indicators. This is especially true in the largest of the four emergency departments, where the team could identify measures with a potential for reducing the actual average lead-time from 3.5 hours to 1.5 hours. Unfortunately, some of the proposed measures still need final approval by the hospital management, which ultimately often depends on internal policy. However, the measures implemented so far have already showed to be very effective, and the average lead-time could be reduced by $17 \%$. As per cost reduction, first effects of a better resource planning could be already measured. As previously outlined, the precarious situation of the large number of access points in the late morning gave rise to adjustment of shift models and redeployment of resources, which already shows positive effects. Moreover, the patient satisfaction perceived by medical and nursing staff has been improved. However, scientific evidence from an actualized patient satisfaction survey is still missing.

4) Lean ought to be implemented holistically in hospital structures and not just inside pilot projects: Eventually, the teams presented the intermediate project results to the managerial board and organized a "roadshow" of presentations to many colleagues from other hospital areas. The encouraging intermediate results and the positive reaction from other colleagues led to an extension of the
Lean initiative from emergency departments to the following wards: medicine, surgery and trauma. In a long-term masterplan, the initiative should be extended to all departments in the hospitals as well as in smaller affiliated clinics. In addition, the results from the Lean initiative were integrated also in several top-level projects regarding the expansion of the hospital structure and of the emergency department. The design of a leaner patient flow and thus a work facilitation for employees and a facilitated orientation for patients and visitors can be achieved. For this purpose, proposals from the Lean initiative are considered in the construction project, and participants from the Lean groups are included in the meetings.

Summarizing positive and negative implications of such a holistic Lean initiative in hospitals leads to the following list of positive implications:

- the definition of patient families leads to a better understanding of the needs of different patients;

- the application of Lean methods requires a reliable triage system, which in turn leads to higher patient safety;

- higher staff satisfaction due to their direct involvement in the design of their work environment;

- higher patient satisfaction due to a reduction of waiting and lead times and more time for direct contact with nursing and medical staff;

- visual management facilitates patient orientation in the hospital, and leads to increased well-being and less interruptions for the nursing and medical staff;

- through in-depth data analysis within the application of the Lean, resources and personnel capacities can be planned more efficiently, thus helping to reduce costs;

- through a redesign of the layout and material or patient flows, non-value adding activities can be reduced and space can be better used;

- transfer processes between different departments can be better coordinated and synchronized;

- a qualification of nursing staff combined with the introduction of standard protocols helps to avoid bottlenecks in the process and therefore reduces the lead-time.

However, some negative or risky implications need also to be taken into account: initially, Lean is seen by many as an industrial method for cost-cutting - thus significant time is needed for initial training, and to achieve the commitment of all the stakeholders; scepticism of single team members may also negatively affect the motivation of all the other team members; visual and concrete partial results need to be achieved relatively quickly to persuade sceptics; it requires the full commitment of the hospital management to initiate a change management process.

The above-mentioned negative or risky implications can be reduced or, hopefully, avoided by a good project management and an accurate information campaign at the beginning of a Lean initiative to limit their risk.

\section{CONCLUSIONS}

Due to its success, Lean is no longer limited to the manufacturing industry, but has been widely implemented within service industry and even public administration. 
Considering the growing need for patient-oriented and efficient health services, Lean has found its way also into hospitals. However, literature review shows that healthcare organisations implement Lean mainly for narrow defined pilot projects, limited to simple methods and tools.

Thus, this research aimed to debate how to optimize patient flows in hospitals in a holistic and systematic way. The research question has then been addressed by discussing the case study of a Lean program carried out in four North Italian hospitals. To overcome the limitations of the Lean experiences reported in literature, an approach for a holistic redesign of the service delivery process in the hospital was chosen based on a comprehensive case study analysis.

The research methodology applied in this research is based on six phases, ranging from a Lean strategy, leadership awareness, Lean training, current state analysis to the definition of the future state and implementation. After having defined suitable patient families with similar process characteristics, the previously trained Lean teams analyzed and optimized the patient flows, elaborating a set of concrete measures. For the redesign of patient flows in the implementation phase, a twelve-step Lean approach was developed. According to a prioritization of these twelve steps, the following were successfully implemented, while others have been postponed. The analysis of takt time resulted in two main blocks with different access rates in the emergency department. This information was important to redefine the needed amount of resources in this time periods. The introduction of the MTS triage system allowed the teams to reduce cycle times and therefore to support a continuous patient flow. The introduction of a queue management system and visual management increases patient satisfaction and reduces the effort of the staff to guide the patients. Workload optimized scheduling allowed an improved capacity utilization of available staff. Nonvalue-adding activities could be reduced thanks to new layout variants to remodel the emergency department. Job enrichment of nursing personnel led to a relief of medical staff. Moreover, access was granted to more flexible shift models, and capacity exchange between different medical stations was improved.

All this contributed to speed up the processes, maximizing value and eliminating waste in the management of patient flows. With regards to performance indicators, measurable improvements can be reported in a reduction of patient lead times by $17 \%$. Furthermore, the results from case study research allowed to confirm the previously defined hypothesis and, thus, to answer the research questions. Therefore, evidence from the research led to conclude that:

- staff satisfaction can be sustainably improved by involving people and interdisciplinary teams in Lean projects;

- a Lean approach increases customer value and patient satisfaction;

- Lead-time from registration to discharge can be minimized by applying Lean approach;

- Lean should be implemented holistically in hospital structures and not just inside pilot projects.

As encouraging as these initial results may be, it must be emphasized that there is still a long way to go to achieve the full implementation of all measures defined by the teams.
Indeed, the programme is still ongoing; it started with a pilot project in the emergency departments, which are subject to this paper, and has now been extended to medical wards, surgery and trauma.

This holistic view is part of a comprehensive strategy to successfully implement Lean principles in healthcare delivery systems. To avoid sub-optimization of narrowly delimited processes and pseudo-innovation [57], the approach presented in this paper allows to keep quality improvement processes sustainable, continuous, and achieving long-term effective results. Indeed, as seen in its application to healthcare delivery, Lean may be applied initially to the process(es) of a single ward; yet, eventually it needs to be expanded to a more complex environment, such as, for example, a ward and a wider overview of patient flow(s) to achieve its full optimization potential.

The application of the Lean methodology described within this case study has different limitations, which must be taken into account when the results are transferred: (a) number of case studies, (b) evaluation of the case study and (c) geographic limitation of the case study. Findings obtained from the consideration of only one case study (a) are not easily transferable to other circumstances. In addition, the development of the methodology as well as the implementation and evaluation of the case study were carried out by the same research team (b). Therefore, the described methodology is to be used and analyzed in other case studies. For further discussion of the approach, it is important to record a larger set of results. This also applies to the specific background of the four North Italian hospitals involved in the case study and their employees (c). Further tests of the systematic approach of the transfer of knowledge need to be performed in different areas of the country; indeed, it is not unlikely that there are significant regional differences that influence the successful application of Lean in healthcare delivery processes.

In conclusion, it is not negligible in the future research also the role of IoT-driven healthcare applications [58] by means of innovative and high technology-based solutions.

\section{REFERENCES}

[1] J. P. Womack and D. T. Jones. Lean Thinking: Banish Waste and Create Wealth in your Corporation. London, UK: Simon and Schuster, 2003.

[2] B. McIntosh, B. Sheppy and I. Cohen, "Illusion or delusion - Lean management in the health sector", International Journal of Health Care Quality Assurance, vol. 27, pp. 482-492, 2014.

[3] A. Giorgetti, C. Cavallini, A. Ciappi, G. Arcidiacono, P. Citti, "A holistic model for the proactive reduction of non-conformities within new industrial technologies", International Journal of Mechanical Engineering and Robotics Research, vol. 6(4), pp. 313-317, 2017.

[4] G. Arcidiacono, C. Calabrese, K. Yang, Leading Processes to Lead Companies: Lean Six Sigma: Kaizen Leader \& Green Belt Handbook. Milan, Italy: Springer Verlag, 2012.

[5] M. Godinho Filho, A. Boschi, A. Freitas Rentes, M. Thurer and T. M. Bertani, "Improving Hospital Performance by Use of Lean Techniques: An Action Research Project in Brazil", Quality Engineering, vol. 27, pp. 196-211, 2016.

[6] P. Nayar, D. Ojha, A. Fetrick and A. T. Nguyen, "Applying Lean Six Sigma to improve medication management", International Journal of Health Care Quality Assurance, vol. 29, pp. 16-23, 2016.

[7] J. De Mast, B. Kemper, R. J. M. M. Does, M. Mandjesb and Y. van der Bijlc, "Process Improvement in Healthcare: Overall Resource Efficiency" Quality and Reliability in Engineering International, vol. 27, pp. 1095-1106, 2011. 
[8] K. C. Van Leeuwen and R. J. M. M. Does, "Quality Quandaries: Lean Nursing”, Quality Engineering, vol. 23, pp. 94-99, 2010.

[9] T. Joosten, I. Bongers and R. Janssen, "Application of Lean thinking to health care: issues and observations", International Journal for Quality in Health Care, vol. 21, pp. 341-347, 2009.

[10] J. P. Womack, D. T. Jones and D. Roos, D. The Machine That Changed the World: The Story of Lean Production - Toyota's Secret Weapon in the Global Car Wars That Is Now Revolutionizing World Industry. New York, USA: Free Press, 1990.

[11] U. Dombrowski and S. Wesemann, "Übertragung der Prinzipien von Ganzheitlichen Produktionssystemen auf das Krankenhaus", Das Krankenhaus, vol. 103, pp. 693-697, 2011.

[12] J. A. Farris, E. M. Van Aken, T. L. Doolen and J. Worley, "Learning from less successful Kaizen events: a case study", Engineering Management Journal, vol. 20, pp. 10-20, 2008.

[13] W. J. Glover, J. A., Farris, E. M. Van Aken and T. L. Doolen, "Critical success factors for the sustainability of Kaizen event human resource outcomes: An empirical study", International Journal of Production Economics, vol. 132, pp. 197-213, 2011.

[14] U. Dombrowski and S. Wesemann, "Paradigmenwechsel in deutschen Krankenhäusern. Ganzheitliches Krankenhaussystem als Lösungsansatz", Das Krankenhaus, vol. 105, pp. 380-385 2013.

[15] B. LariSemnani, R. Mohebbi Far, E. Shalipoor and M. Mohseni, "Using creative problem solving (TRIZ) in improving the quality of hospital services", Global Journal of Health Science, vol. 7, pp. 8897, 2015.

[16] G. Arcidiacono and L. Bucciarelli, "TRIZ: Engineering Methodologies to Improve the Process Reliability", Quality and Reliability Engineering International Journal, vol. 32, pp. 25372547, 2016.

[17] G. Arcidiacono, A. Giorgetti and M. Pugliese, "Axiomatic Design to improve PRM airport assistance", in Proc. ICAD 2015, 9th International Conference on Axiomatic Design, 2015, pp. 106-111.

[18] M. Baker, I. Taylor and A. Mitchell. Making Hospitals Work. Goodrich, UK: Lean Enterprise Academy Limited, 2009.

[19] H. De Koning, J. P. S. Verver, J. Van Den Heuvel, S. Bisgaard and R. J. Does, "Lean Six Sigma in healthcare", Journal for Healthcare Quality, vol. 28, pp. 4-11, 2006

[20] O. Groene, D. Botje, R. Suñol, M. A. Lopez and C. Wagner, “A systematic review of instruments that assess the implementation of hospital quality management systems", International Journal for Quality in Health Care, vol. 25, pp. 525-541, 2013.

[21] B. Kemper, J. de Mast and M. Mandjes, "Modeling process flow using diagrams", Quality and Reliability Engineering International, vol. 26, pp. 341-349, 2010.

[22] B. Kemper and J. de Mast, "Measurement Plans for Process Flow Improvement in Services and Health Care", Quality Engineering, vol. 25, pp. 437-450, 2013.

[23] S. Bhasin and P. G. Burcher, "Lean viewed as a philosophy", Journal of Manufacturing Technology Management, vol. 17, pp. 56-72, 2006.

[24] M. Graban. Lean Hospitals. New York, USA: Taylor \& Francis, 2009.

[25] G. Arcidiacono, J. Wang and K. Yang, "Operating room adjusted utilization study”. International Journal of Lean Six Sigma, vol. 6, pp. 111-137, 2015.

[26] D. T. Matt, V. M. Franzellin and E. Rauch, "Lean Hospital - Mit Motivation und Methode zum schlanken Krankenhausbetrieb", Das Krankenhaus, vol. 106, pp. 538-542, 2014

[27] D. T. Matt, M. Siller and M. Prantl, "Patientenorientierte und effiziente Notfallversorgung durch Lean Hospital in Südtiroler Krankenhäusern”, Das Krankenhaus, vol. 107, pp. 555-561, 2015.

[28] D. T. Matt, E. Rauch and V. M. Franzellin, "An Axiomatic Design based approach for the patient-value oriented design of a sustainable Lean Healthcare System", International Journal of Procurement Management, vol. 8, pp. 66-81, 2015.

[29] X. Xie and M. A. Lawley, "Operations research in healthcare", International Journal of Production Research, vol. 53, pp. 71737176, 2015.

[30] J. Li, C. T. Papadopoulos and L. Zhang, "Continuous improvement in manufacturing and service systems", International Journal of Production Research, vol. 54, pp. 6281-6284, 2016.

[31] J. H. Sanders and T. Karr, ,Improving ED specimen TAT using lean six sigma”. International Journal of Health Care Quality Assurance, vol 28, pp. 428-440, 2015.

[32] A. Abdelhadi, "Investigating emergency room service quality using lean manufacturing". International Journal of Health Care Quality Assurance, vol 28, pp. 510-519, 2015.
[33] K. Al-Hyari, S. Abu Hammour, M. K S. Abu Zaid and M. Haffar, "The impact of Lean bundles on hospital performance: does size matter?". International Journal of Health Care Quality Assurance, vol 29, pp. 877-894, 2016.

[34] P. Simons, H. Backes, J. Bergs, D. Emans, M. Johannesma, M Jacobs, W. Marneffe and D. Vandijck, "The effects of a lean transition on process times, patients and employees". International Journal of Health Care Quality Assurance, vol. 30, pp. 103-118, 2017.

[35] P. Hwang, D. Hwang and P. C. Hong, "Lean practices for quality results: a case illustration", International Journal of Health Care Quality Assurance, vol. 27, pp. 729-741, 2014.

[36] B. Kollberg, J. J. Dahlgaard and P. O. Brehmer, "Measuring Lean initiatives in health care services: issues and findings", International Journal of Productivity and Performance Management, vol. 56, pp. 7-24, 2007.

[37] L. Brandao de Souza, "Trends and approaches in Lean healthcare", Leadership in Health Services, vol. 22, pp. 121-139, 2009

[38] J. L. Dellifraine, J. R. Langabeer and I. M. Nembhard, "Assessing the evidence of Six Sigma and Lean in the health care industry", Quality Management in Health Care, vol. 19, pp. 211-225, 2010.

[39] N. Burgess and Z. Radnor, "Evaluating Lean in healthcare". International Journal of Health Care Quality Assurance, vol. 26, pp. 220-235, 2013.

[40] P. Dannapfel, B. Poksinska and K. Thomas, "Dissemination strategy for Lean thinking in health care". International Journal of Health Care Quality Assurance, vol 27, pp. 391-404, 2014.

[41] S. Knapp, "Lean Six Sigma implementation and organizational culture". International Journal of Health Care Quality Assurance, vol 28, pp. 855-863, 2015.

[42] M. Crema and C. Verbano, "Investigating the connections between health lean management and clinical risk management: insights from a systematic literature review". International Journal of Health Care Quality Assurance, vol 28, pp. 791-811, 2015.

[43] S. Deblois and L. Lepanto "Lean and Six Sigma in acute care: a systematic review of reviews", International Journal of Health Care Quality Assurance, vol 29, pp. 192-208, 2016.

[44] T. Katz-Navon, E. Naveh and Z. Stern, "The moderate success of quality of care improvement efforts: three observations on the situation", International Journal for Quality in Health Care, vol. 19 pp. 4-7, 2007.

[45] M. Harrison, K. Henriksen and R. Hughes, "Improving the health care work environment: a sociotechnical systems approach", Joint Commission journal on quality and patient safety, vol. 33, pp. 3-6, 2007.

[46] J. Moraros, M. Lemstra and C. Nwankwo, "Lean interventions in healthcare - do they actually work? A systematic literature review", International Journal for Quality in Health Care, vol. 28, pp. 150$165,2016$.

[47] T. N. Goh, "Six Triumphs and Six Tragedies of Six Sigma", Quality Engineering, vol. 22, pp. 299-305, 2010.

[48] N. V. K. Jasti and R. Kodali, "Lean production: literature review and trends", International Journal of Production Research, vol. 53, pp. $867-885,2015$.

[49] A. Taylor, M. Taylor and A. McSweeney, "Towards greater understanding of success and survival of Lean systems", International Journal of Production Research, vol. 51, pp. 66076630, 2013.

[50] G. Arcidiacono, N. Costantino and K. Yang, "The AMSE Lean Six Sigma Governance Model", International Journal of Lean Six Sigma, vol. 7, pp. 233-266, 2016

[51] G. C. Niemeijer, R. J. Does, J. de Mast, A. Trip and J. van den Heuvel, "Generic project definitions for improvement of health care delivery: a case-based approach", Quality Management in Health Care, vol. 20, pp. 152-164, 2011.

[52] G. Arcidiacono, D.T. Matt, E. Rauch, "Axiomatic Design of a Framework for the Comprehensive Optimization of Patient Flows in Hospitals", Journal of HealthCare Engineering, vol. 2017, Article ID 2309265, 9 pp., 2017.

[53] R. K. Yin. Case study research: Design and methods. Thousand Oaks, USA: Sage publications, 2013.

[54] P. Mazzocato, R. J. Holden, M. Brommels, H. Aronsson, U. Bäckman, M. Elg and J. Thor, "How does lean work in emergency care? A case study of a lean-inspired intervention at the Astrid Lindgren Children's hospital, Stockholm, Sweden", BMC Health Services Research, vol. 12, pp. 28, 2012. 
[55] W. Ulhassan, C. Sandahl, H. Westerlund, P. Henriksson, M. Bennermo, U. von Thiele Schwarz and J. Thor, ,Antecedents and characteristics of lean thinking implementation in a Swedish hospital: a case study", Quality Management in Healthcare, vol. 22, pp. 48-61, 2013.

[56] S. L. Dong, M. J. Bullard, D. P. Meurer, S. Blitz, A. Ohinmaa, B. R. Holroyd and B. H. Rowe, "Reliability of computerized emergency triage”, Academic Emergency Medicine, vol. 13, pp. 269-275, 2006.
[57] K. Walshe, "Pseudoinnovation: the development and spread of healthcare quality improvement methodologies", International Journal for Quality in Health Care, vol. 21, pp. 153-159, 2009.

[58] N. Scarpato, A. Pieroni, L. Di Nunzio, F. Fallucchi, "E-health-IoT Universe: A Review", International Journal on Advanced Science Engineering Information Technology, vol. 7, pp. 2328-2336, 2017. 